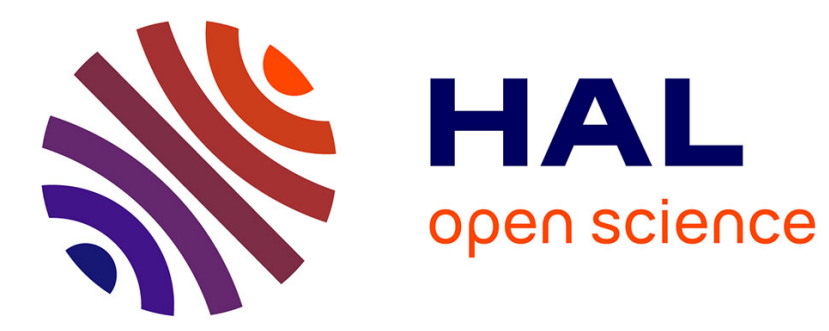

\title{
Le désir de mobilité et l'automobilisme. Pour une analyse systémique de la vitesse automobile dans l'espace rural : le cas du département du Rhône et de la région de Québec \\ Étienne Faugier
}

\section{To cite this version:}

Étienne Faugier. Le désir de mobilité et l'automobilisme. Pour une analyse systémique de la vitesse automobile dans l'espace rural: le cas du département du Rhône et de la région de Québec. RTS - Recherche Transports Sécurité, 2015, Usages de la vitesse, 2015 (01), pp.7-24. 10.4074/S0761898015001028 . hal-01670606

\section{HAL Id: hal-01670606 https://hal.science/hal-01670606}

Submitted on 21 Dec 2017

HAL is a multi-disciplinary open access archive for the deposit and dissemination of scientific research documents, whether they are published or not. The documents may come from teaching and research institutions in France or abroad, or from public or private research centers.
L'archive ouverte pluridisciplinaire HAL, est destinée au dépôt et à la diffusion de documents scientifiques de niveau recherche, publiés ou non, émanant des établissements d'enseignement et de recherche français ou étrangers, des laboratoires publics ou privés. 


\title{
Le désir de mobilité et l'automobilisme. Pour une analyse systémique de la vitesse automobile dans l'espace rural : le cas du département du Rhône et de la région de Québec
}

\author{
Mobility's Desire and Automobilism. Systemic Analyse of Automobile Speed in Rural Area: \\ Case Studies of the Department of Rhône and Québec Region's
}

\author{
Étienne Faugier \\ (C) IFSTTAR et Éditions NecPlus 2015
}

Résumé La mobilité par l'automobilisme a conduit à une véritable révolution. Révolution du rapport au temps, révolution du rapport à l'espace : soit une révolution de la vitesse. Dans cet article, nous avançons l'idée selon laquelle ce sont les mobilités individuelles reprises par les pouvoirs publics qui ont initié l'essor du système de la vitesse automobile. Avec une approche historique, nous mettons en avant la manière dont s'est constitué la vitesse automobile depuis les usages pionniers, en passant par la constitution d'une culture du mouvement et enfin à l'élaboration d'un réseau routier amenant aux prémices d'une nouvelle vitesse : la grande vitesse, celle des autoroutes.

Mots-clés vitesse $\cdot$ automobile $\cdot$ histoire contemporaine . mutations

Summary Mobility by motoring led to a revolution. Revolution about time, revolution about the relation to space: either a speed's revolution. In this article, we put forward the idea that it is the individual mobility followed by the government that initiated the development of the system of automobile speed. With a historical approach, we highlight how the motor car was set up speed from the pioneer's uses, through the establishment of a culture of movement and finally the development of a road network leading to the beginnings of a new speed: high speed through the highways.

Keywords speed $\cdot$ motor car · contemporary history · changes

Étienne Faugier $(\bowtie)$

Institut d'histoire, Université de Neuchâtel

88 rue Antoine Charial, 69003, Lyon

e-mail : etienne.faugier@gmail.com

\section{Introduction}

Les « mobilités concrètes », entendues les déplacements physiques, sont la base de l'individu [1]. Avec l'essor de l'automobile dès la fin du $\mathrm{XIX}^{\mathrm{e}}$ siècle, les individus acquièrent un nouveau moyen de déplacement. À l'inverse du transport ferroviaire qui propose une mobilité collective et limitée par un réseau hiérarchisé [2], l'automobile permet un déplacement individuel avec une possibilité de voyage plus ample. Ce mode et sa massification procurent progressivement un nouveau rapport à l'espace et au temps ; soit une nouvelle vitesse : la vitesse automobile.

On peut se demander dans quelle mesure le désir de mobilité des individus par le biais de l'automobile mène à l'essor du système de la vitesse automobile dans le Rhône et la région de Québec entre 1919 et 1961. Notre hypothèse est la suivante : le désir de mobilité des pionniers de l'automobile a stimulé l'essor du système de la vitesse automobile dont la constitution réside principalement entre les mains des pouvoirs publics. Une véritable révolution de la mobilité par l'automobilisme a conquis progressivement les individus depuis les élites jusqu'à l'État en passant ensuite par les masses [3].

Après avoir explicité le contexte et la méthodologie entourant notre étude, nous traiterons tout d'abord les différents usages et appropriations de l'automobile au sein des deux sociétés et la manière dont ils s'imbriquent au sein du système de la vitesse automobile. Puis, nous verrons la progressive construction d'une structuration mentale du territoire chez les populations qui accroît la capabilité des individus à se déplacer sur le territoire. Enfin, nous étudierons l'essor de l'infrastructure, élément indispensable au déplacement automobile. 


\section{Contextualiser et délimiter l'étude}

\section{Choix du terrain d'analyse}

La région de Québec n'étant pas un territoire politiquement et administrativement définie, en utilisant l'historiographie [4], nous l'avons défini comme regroupant les comtés de Portneuf, de Montmorency, de l'île d'Orléans et la ville de Québec. Pour le cas du département du Rhône, nous avons sélectionné l'espace administratif délimité par le politique durant le $\mathrm{XX}^{\mathrm{e}}$ siècle. Pour ces deux territoires, nous concentrons notre attention en marge de l'espace urbain, en direction de l'espace rural, car les mutations intervenues avec l'irruption de l'automobilisme dans le monde rural excèdent de beaucoup celles survenues dans le monde urbain [5].

Mettre en relation le département du Rhône et la région de Québec a conduit à évaluer les ressemblances et les différences entre ces deux territoires. Il s'avère qu'ils demeurent relativement similaires quant à la structuration géographique autour d'un pôle urbain dominant, la répartition de la population à proximité du fleuve et l'économie. Ils ont pour points communs la culture francophone, le démarrage de l'exode rural à la fin $\mathrm{XIX}^{\mathrm{e}}$-début $\mathrm{XX}^{\mathrm{e}}$ siècle, la mutation de l'agriculture s'effectue de manière quasi identique au cours du $\mathrm{XX}^{\mathrm{e}}$ siècle, la domination de l'industrie laitière y est observable, l'utilisation du cheval dans les campagnes disparaît vers les années 1960 et les grands projets autoroutiers commencent autour de la fin des années 1950 et au début des années 1960. Pour ce qui est des différences, le Rhône est un département particulièrement urbanisé et industrialisé - Lyon est le siège de multiples entreprises et industries automobiles - contrairement à la région de Québec qui s'équipe en automobiles grâce à ses voisins tels que l'Ontario et les États-Unis, et possède une industrie diversifiée. L'influence américaine au Québec se ressent dès les années 1909-1910 avec la présence du modèle $T$ de Ford qui pénètre dans les campagnes dans les années 1920 ; dans les campagnes du Rhône, l'accessibilité aux véhicules à moteur est plus tardive puisqu'elle intervient après la Seconde Guerre mondiale avec le Plan Marshall de 1947. Le Rhône subit les deux guerres mondiales, tandis que le Québec ne souffre d'aucun dommage matériel même s'il participe à l'effort de guerre et la consommation automobile ne connaît pas de réel ralentissement. Enfin, la région de Québec se caractérise par un climat hivernal particulièrement long et froid, tandis que le Rhône connaît moins ces difficultés météorologiques. L'ensemble de ces divers éléments permet une mise en relation fertile.

\section{Un âge de la vitesse automobile?}

La période arrêtée - 1919-1961 - est pertinente, car les bornes choisies évoquent des moments décisifs dans l'histoire de chacun des territoires en ce qui concerne l'essor du système automobile.

Pour ce qui est du Rhône, les deux dates retenues sont des ruptures. En 1919, des efforts faits pour promouvoir la vitesse automobile et plus particulièrement son système - une circulaire administrative définit le type de panneaux indicateurs à placer sur les routes à l'intention des usagers de la route. En 1961 est créée de la Société de l'Autoroute Paris-Lyon (Autoroute A6) qui a pour but de réaliser l'autoroute reliant Paris à Lyon qui inaugure l'ère de la grande vitesse sur route.

Dans le cas de la région de Québec, la première borne témoigne de la prise de conscience du poids quantitatif de l'automobile dans la Province avec l'enregistrement obligatoire au bureau du Trésorier provincial des automobiles par l'État provincial. La seconde indique la naissance d'une nouvelle ère dans le transport routier avec l'essor de la grande vitesse par les autoroutes avec la création de l'Office des autoroutes du Québec par la loi du 25 mai 1961.

\section{L'approche méthodologique}

\section{L'histoire croisée et le croisement de points de vue}

Pour analyser les deux territoires l'un avec l'autre et l'un par rapport à l'autre, plusieurs approches méthodologiques s'offrent à nous.

L'histoire comparée semble être le premier mouvement. Pourtant, ce chemin nous aurait amené à faire le constat binaire des ressemblances et des différences entre les deux espaces ; élément qu'il fallait dépasser [6]. Les études relatives aux transferts ou aux circulations culturelles n'ont pas lieu d'être sollicitées puisqu'ils sont pertinents lors de travaux diachroniques alors que nous privilégions la synchronie [7]. De la même manière, l'histoire connectée ne convient pas puisque les contacts entre les deux territoires sont presque absents [8]. Ces différentes méthodes ne nous satisfaisant pas complètement, nous nous sommes tournés vers une autre approche.

L'histoire croisée est la méthode que nous avons retenue et plus spécifiquement le croisement des points de vue [9]. L'automobilisme et ses impacts au sein des deux territoires étant dissemblables, nous avons fait le choix de surimposer un concept fédérateur ou une équivalence fonctionnelle qui permet de croiser équitablement les deux objets de recherche 
[10]. Ce dénominateur commun requiert d'être défini de manière flexible pour prévenir de la malléabilité des deux terrains d'étude et à la fois d'une certaine rigidité afin de ne pas devenir inutile. Le concept, construit par nos soins à l'aide de l'historiographie contemporaine, est celui de la vitesse.

\section{État de l'art de la vitesse}

Le concept de vitesse se trouve mobilisé de diverses manières dans plusieurs disciplines. Les littéraires et quelques historiens l'ont utilisé pour analyser les mutations produites par l'essor du train et de l'automobile dans les arts et en relation avec la notion de modernité [11]. Les urbanistes et géographes l'ont appréhendé comme un facteur d'aménagement - positif ou négatif - de la ville et du territoire [12]. L'histoire des records de vitesse a été un champ d'investigation pour les historiens des techniques [13]. Peu de spécialistes de l'histoire des transports ont abordé la vitesse, car cette dernière pose des difficultés quant à son apprivoisement dans les sources susceptibles d'être consultées [14]. Christophe Studeny, dans une étude pionnière en histoire, s'est intéressé à la naissance et à l'essor du concept de vitesse dans l'imaginaire de la population française depuis le XVIII ${ }^{\mathrm{e}}$ siècle avec les coches et la Poste jusqu'au $\mathrm{XX}^{\mathrm{e}}$ siècle avec l'avion. Il prend donc le parti d'analyser l'accélération de la vitesse en France : soit les changements de registre de la vitesse [14]. Cette approche culturelle se concrétise par une notule dans le Dictionnaire d'histoire culturelle où Vincent Guigueno défend l'idée d'une approche systémique du phénomène [15]. Ce dernier, dans un article récent, lie vitesse et systèmes de transport et propose ainsi la notion de paysages de transport en s'adossant au travail de Marc Desportes [16]; notion que l'on retrouve dans l'ouvrage collectif dirigé par Christof Mauch et Thomas Zeller [17].

En nous inspirant des différents travaux évoqués, nous proposons de définir la vitesse comme un système logique et cohérent ou un "socio-système » [18] qui s'appuie sur un mobile - le véhicule à moteur -, une infrastructure - la route -, une culture et des pratiques de mobilité. Il est pertinent d'étudier ces éléments spécifiquement, car ils sont interdépendants et concomitants : l'automobile a besoin de la route et la route est la raison d'être de l'automobile ; l'individu a besoin d'une culture automobile et d'un véhicule pour devenir automobiliste. Rappelons que le système de la vitesse automobile se construit largement grâce aux systèmes de vitesse des autres modes de transport : celle-ci emprunte à la vitesse vélocipédique le regroupement en modèle associatif, à la vitesse hippomobile l'infrastructure économique soutenant le système et cohabite relativement avec la vitesse ferroviaire. Il est donc pertinent d'appréhender le cas de l'automobilisme, car il est à la fois un vecteur de la vitesse [19] et il illustre cette dernière - avec le mouvement futuriste par exemple [20].

L'analyse du système de la vitesse est impossible à faire dans son intégralité dans le cadre d'un article. Dans ces conditions, l'aspect technique - soit l'évolution de la puissance motrice et des performances des véhicules à moteur -, bien qu'important, n'a pas été retenu dans notre analyse, car d'une part il a été traité par les historiens des techniques et les historiens économistes [21] et d'autre part, il ne suffit pas à expliquer la vitesse automobile ; elle réside dans une histoire politique, économique et culturelle. Par ailleurs, l'étude de la circulation des savoirs relatifs à l'automobilisme a été délaissée puisqu'elle a fait l'objet de recherches [22].

En prenant le parti de la notion de vitesse automobile plutôt que celui de système automobile, nous souhaitons mettre l'accent sur le mouvement et la mentalité qui l'accompagne - le rapport de l'espace parcouru ramené au temps - en montrant qu'il s'appuie sur divers éléments qui, pris un à un, ne sont pas forcément mobiles, mais ensemble permettent la mobilité : en soi, la normalisation n'a pas de vitesse par exemple. De fait, nous n'entendons pas cette notion au sens de rapidité ou d'accélération : la vitesse automobile, si elle s'exprime souvent en $\mathrm{km} / \mathrm{h}$, ne peut être uniquement ramenée qu'à cet indice quantitatif.

Voyons plus en détail ce qu'il en est de la mobilité des individus avec l'automobile au sein des deux territoires prédéfinis.

\section{Mettre en pratique la vitesse : la mobilité par l'automobilisation}

Avec l'essor de l'automobilisation, les individus s'approprient ce nouveau moyen de transport pour combler leurs besoins. Les usages de la vitesse automobile sont multiples et proposer une vision totale de ces derniers est impossible, nous nous sommes donc limités à quelques-uns.

\section{L'appropriation de la vitesse automobile : le temps des pionniers ruraux}

Au sein de la société rurale française, le docteur est l'un des premiers individus à acquérir une automobile. Elle lui permet d'être mobile bien plus rapidement qu'avec le cheval ; ce faisant, il devient l'un des promoteurs de l'automobile auprès des populations rurales. Parallèlement, l'Union nationale des associations de tourisme, en collaboration avec la Croix-Rouge, entreprend de former quelques professionnels - les restaurateurs, les pompistes - aux premiers soins et il fait installer sur le bord des routes des grands itinéraires des postes de secours localisés aux endroits dangereux durant les années 1920-1930. On y 
trouve du matériel de premiers soins, soit un brancard, des couvertures, un nécessaire médical et un personnel médical assermenté. Au sein du département du Rhône, les postes se localisent au nord du département sur la route nationale 6 , la plus empruntée, car elle relie le nord au sud de la France en passant par Lyon. Plusieurs communes accueillent ces postes de secours : Saint-Jean-d'Ardières, Saint-Georges, Villefranche-sur-Saône, Anse, Lissieu et Champagne. On installe ainsi les postes de secours là où ils sont susceptibles d'être le plus demandés. Il y a donc progressivement une hiérarchisation du réseau routier qui se construit par le déploiement quantitatif des mobilités individuelles. Cet exemple illustre parfaitement l'ancrage qui est fait entre la mobilité des individus et le territoire : des points de contact potentiellement nécessaires durant le voyage se matérialisent.

Dans la région de Québec, le médecin est l'un des premiers possesseurs d'automobile. Elle lui permet de ne plus dépendre du cheval et elle est plus rapide que l'équidé. Les conditions climatiques obligent le praticien à conserver l'équidé comme moyen de transport, même si certains adaptent leur véhicule à l'hiver : G. Desrosiers, médecin de campagne, construit une voiture chauffée [23]. L'innovation technologique s'effectue aussi par le bas, soit par les individus [24]. C'est d'ailleurs dans la banlieue de Québec qu'est créée, à la fin des années 1930, l'autoneige par Joseph Armand Bombardier. Durant les années 1950-1960, l'entreprise Bombardier interpelle les professionnels de la santé, car ce nouveau véhicule surmontant l'hiver peut les satisfaire ; ce qui est le cas. On ne trouve pas comme dans le Rhône de structuration du territoire par l'automobile médicale du fait du morcellement du territoire québécois.

L'usage médical de l'automobilisme influence profondément l'aménagement du territoire et le rapport à l'espace. Dans ce cas précis, les mobilités individuelles orientent la constitution du système de la vitesse automobile par les pouvoirs publics.

L'insertion de la vitesse automobile au sein de la collecte de lait bouleverse ces deux bassins laitiers importants. Les relations ville-campagne sont redéfinies puisque cet aliment, issu des campagnes, nourrit la population de la ville sous différentes formes : le lait en bouteille, le beurre ou le fromage.

Jusque dans les années 1920-1930, le lait, une fois collecté, est redistribué par l'intermédiaire des multiples gares ferroviaires lyonnaises - par exemple les gares de Vaise, Croix-Rousse, Perrache, Cordeliers, Brotteaux, Villeurbanne - ou au centre de pasteurisation le plus proche. Pour autant, le transport ferroviaire présente plusieurs limites : les bidons subissent des pertes ou sont l'objet de vol, les horaires fixes et rigides et la relative accessibilité des gares ne satisfont pas tous les industriels et leurs clientèles. Après la Première Guerre mondiale, le transport de lait par camions croît du fait des progrès techniques et des atouts des véhicules motorisés. Les industriels, comme Renault durant les années 1930, élaborent des véhicules motorisés pour les compagnies laitières telles que la Société Laitière Moderne de Lyon [25]. Grâce au progrès technique, les camions et les camionnettes deviennent isothermes puis réfrigérés [25]. Ces véhicules élargissent l'aire des bassins laitiers en allant collecter le lait plus loin que ne le fait le chemin de fer. De plus, ils suppriment les intermédiaires entre les producteurs et leurs clients. Et enfin, les dépôts de quartier, répartis dans toute la ville, proposent une nouvelle structuration de l'espace urbain lyonnais.

Dans la région de Québec, le transport du lait depuis les fermes des cultivateurs jusqu'aux coopératives s'effectue par cheval et par carriole tractée par des chiens. Cette mobilité est lente du fait de la distance entre les exploitations et les beurreries et des contraintes de la traction hippomobile. De fait, les exploitants se regroupent de manière à alterner les déplacements pour ne pas perdre une demi-journée de travail ou déposent leur lait en bordure de route dans des bidons soumis à une réglementation stricte. Cette situation perdure jusqu'à l'apparition des camions et camionnettes réfrigérés dans les années 1950-1960. Dans la région de Québec, la présence majoritaire de grandes laiteries ralentit fortement l'essor quantitatif des coopératives dans le domaine de l'industrie laitière [4].

Au sein des deux espaces, la vitesse automobile s'adapte au besoin des individus, modifie les pratiques de mobilité, réorganise l'industrie laitière pour proposer un nouveau rapport à l'espace et au temps.

\section{Lier l'information à la vitesse automobile}

Le service postal français transforme, dès le début du siècle, l'automobile pour acheminer le courrier et les marchandises. Le service de la Poste automobile rurale (PAR), inauguré au début des années 1920, lutte contre l'isolement rural puisque sont desservies les communes dépourvues de bureau de poste et abritant moins de 2500 habitants. Cinq circuits sont créés durant les années 1920-1930 pour répondre à l'absence ou le faible taux de la motorisation de plusieurs communes, à cause de faiblesse structurelle relative au réseau routier - Condrieu, Givors, Mornant, Sainte-Foy-L'Argentière et Beaujeu. Cette prestation réaménage, d'une part, elle la temporalité de la vie rurale - les nouvelles sont attendues à heures fixes - et d'autre part, les relais postaux institués tissent des liens économiques et sociaux. Quatre autres circuits sont ouverts dans les années 1960 - Thizy, Larajasse, Saint-Symphorien-sur-Coise et Cours. Cependant, avec l'essor des voitures populaires, les postiers font désormais leurs tournées rurales en 4 CV à partir de 1947, en fourgonnette $2 \mathrm{CV}$ Citroën et en Renault 4. À partir de 1952-1953, la généralisation de la motorisation est décidée en 1954 ; c'est le début de la fin du service de la PAR [26]. 
La PAR met en évidence les relations qui existent entre le mobile, l'usager et le réseau et le territoire. Ce service postal étant une originalité française, il n'a pas d'équivalent pour la région de Québec. La distribution du courrier prend diverses formes : à Lévis, un service régulier de messagerie par camion et tramway dessert Lauzon, Lévis, Saint-David, Saint-Télesphore et Saint-Romuald. La généralisation de l'automobile et de l'autoneige postale intervient dans les années 1960 et s'appuie aussi sur des infrastructures économiques telles le bureau de poste.

L'institution de nouveaux points de contact, de nouvelles pratiques de mobilité, de sociabilités renouvelées fait apparaître une nouvelle hiérarchisation territoire. Le maillage spatial est redessiné plus finement par la vitesse automobile à partir des usages individuels et collectifs des véhicules motorisés : à la fois pratiquement par l'intermédiaire du réseau routier et mentalement par l'essor d'une culture automobile.

Les usages de la vitesse sont relativement proches entre les deux territoires. Néanmoins, une structuration $\mathrm{du}$ territoire se fait jour plus fortement dans le Rhône $\mathrm{du}$ fait de l'historicité du réseau routier plus maillé que dans la région de Québec. Les pionniers automobiles et les différents usagers sont à l'origine de mobilités et d'innovations techniques modernes qui modifient le rapport au temps et à l'espace. Parallèlement se déploie par divers canaux une culture de la vitesse automobile.

\section{De nouvelles pratiques de mobilité amenant à une nouvelle conception mentale du territoire}

La vitesse automobile se construit avec une nouvelle structuration mentale du paysage. Les conducteurs acquièrent une façon de penser et d'appréhender le territoire. Cela passe par l'élaboration de littératures de l'automobilisme, qui une fois de plus est entreprise par les pionniers de l'automobilisme puis reprise et renforcée par les pouvoirs publics. Deux mouvements sont à l'œuvre : la culture de la vitesse automobile organise mentalement le territoire et elle offre une image partielle de la réalité.

\section{La vitesse automobile au fil des pages : \\ la constitution d'une culture des déplacements automobiles}

\section{Les clubs-automobile : \\ initiateur de la bibliothèque du savoir automobile}

Les premiers automobilistes se regroupent sous la forme d'association : les clubs automobiles. À Lyon, l'ACR
(Automobile-Club du Rhône) est créé en 1902, tandis que dans la région de Québec, le CAQ voit le jour en 1912 : ce décalage s'explique par l'essor industriel entourant le cycle et l'automobile dans le département du Rhône contrairement à la région de Québec. Ces clubs promeuvent l'automobilisme et défendent leurs membres : cela passe par le Bulletin de l'Automobile-Club du Rhône que l'ACR publie dès 1902 et le CAQ édite dès 1912 Motoring Magazine.

Regroupent des informations relatives aux travaux de voirie effectués et envisagés, à l'état des routes, à la signalisation routière apposée, ces deux revues font prendre conscience aux lecteurs-automobilistes du déploiement de l'infrastructure dont ils dépendent : la route. Elles informent de la législation concernant tous les véhicules et tous les usagers de la route confondus à l'échelle régionale, nationale et internationale et de son évolution. Les deux clubs disposent d'un salon de lecture et d'une bibliothèque, mis à la disposition des membres, composés d'ouvrages spécifiques à l'automobile et aux transports. Ces données collectées élargissent le territoire mental des automobilistes-membres ; Cependant, en parallèle, les associations s'adressent aussi à un public plus large par le biais d'autres démarches.

Les deux clubs publient des guides touristiques annuels qui synthétisent les éléments présents dans leur revue afin de faciliter le déplacement des touristes automobiles. On y retrouve donc des cartes routières, des index pointant les municipalités, les hôtels et les garages, quelques photographies et le pointage des richesses touristiques et naturelles. Cette littérature permet aux membres des clubs et aux automobilistes de se déplacer plus aisément sur le territoire et d'avoir une conscience élargie de leur environnement. Le CAQ se démarque de l'ACR, du fait de la proximité de la nation asphaltée américaine, par l'inauguration à Québec en 1923 d'un office de tourisme qui informe les touristes par téléphone, par écrit ou bien simplement lors de rencontres concrètes.

Les associations automobiles fournissent le substrat théorique de la vitesse : soit les connaissances relatives à la capabilité des conducteurs à bouger dans l'espace. Les membres des clubs puis les automobilistes appréhendent mentalement le territoire par les cartes routières, l'iconographie et les articles. D'autres acteurs participent de la production et de l'essor de la culture de la vitesse.

\section{Cultiver le phénomène automobile : la pluralité des écrivains}

La Fédération des syndicats d'initiative de la vallée du Rhône produit des fascicules touristiques valorisant les infrastructures et les points touristiques. Elle s'adresse au 


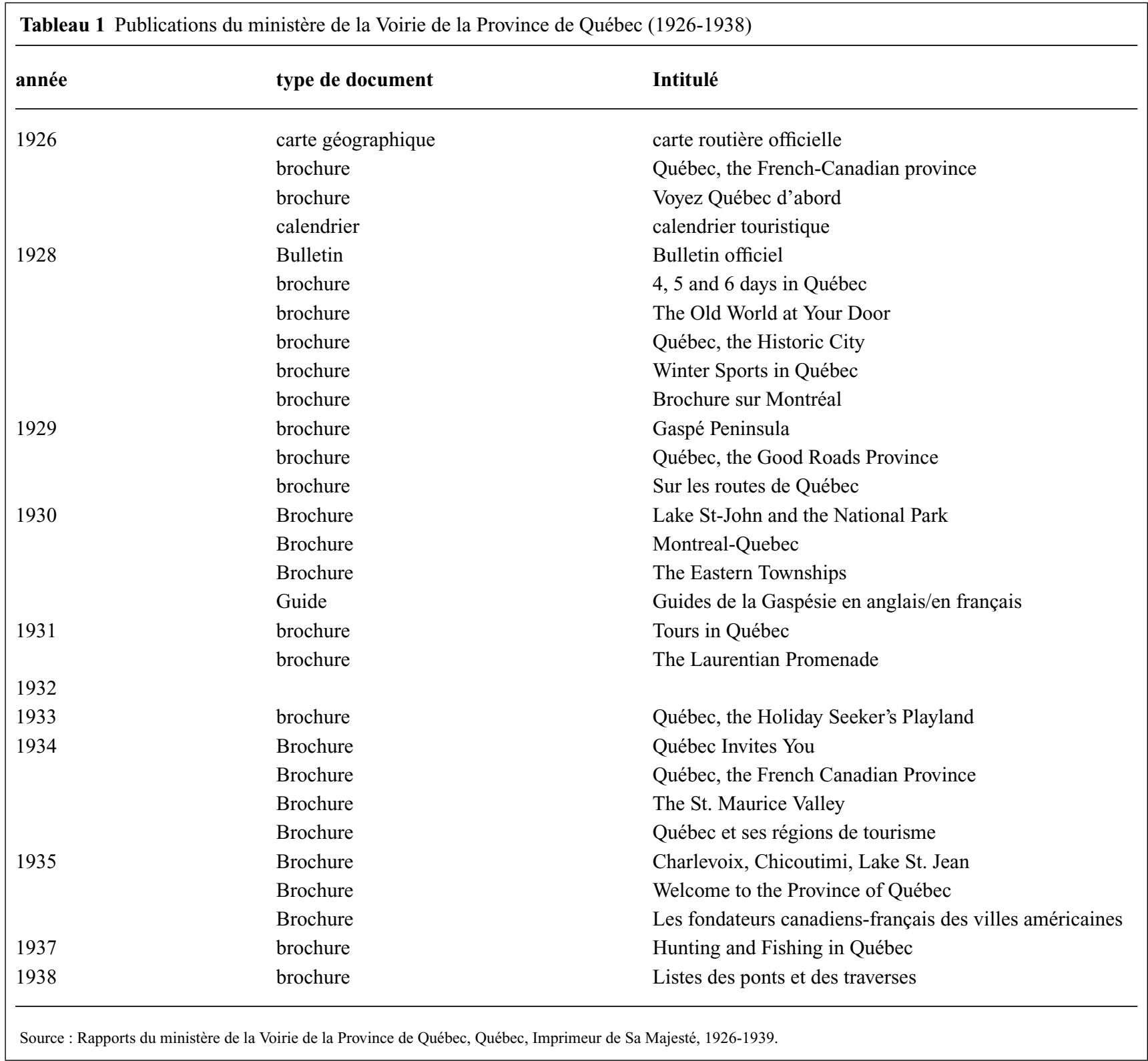

grand public intéressé par les déplacements en automobile, en autocar ou en train [27].

À l'opposé, le ministère de la Voirie de la Province de Québec promeut spécifiquement les littératures automobiles (tableau 1).

Ces différentes publications, rééditées annuellement, structurent la Province en régions touristiques avec leur identité propre [28], contribuent à l'étalonnage des distances en fonction du ratio $\mathrm{km} / \mathrm{h}$ et participent de la cartographie mentale du territoire.

Ces deux acteurs, en promouvant le tourisme automobile, contribuent à l'essor de la culture de la vitesse. Les associations en passant par les syndicats jusqu'aux pouvoirs publics se partagent la propagande automobile ; au Québec, l'État s'impose comme promoteur principal du tourisme et relègue les associations automobiles en seconde position. Ces littératures n'encouragent pas la célérité durant le voyage au contraire, mais sert de cadre de référence pour les automobilistes en stipulant les lieux touristiques, les infrastructures économiques automobiles (garages, hôtels), la distance et la durée entre différents points de contact. Cette culture automobile est indispensable au système de la vitesse pour fonctionner adéquatement. Ce n'est cependant pas la seule : la littérature législative importe aussi. 
Maîtriser la puissance des véhicules à moteur : l'élaboration de la normalisation

\section{Les tâtonnements de la législation automobile : les spécificités du monde rural}

$\mathrm{Au}$ sein des deux territoires, la législation automobile se construit similairement même si la chronologie diffère (tableau 2). Premier texte juridique automobile, le décret de 1899 apparaît en France sept ans plus tôt qu'au Québec du fait de la place de l'automobile. Ces deux textes enjoignent les automobilistes de respecter les codes de conduite et les informent des punitions en cas de non-respect de ces derniers et de posséder un véhicule conforme aux attentes définies par l'État - enregistrement officiel, plaques d'immatriculation, phares. $\mathrm{Du}$ fait du poids du monde rural français sur la société, l'obligation d'être éclairée ne s'applique pas aux véhicules agricoles lorsqu'ils circulent entre la ferme et les champs et lors du transport de récolte. Par ailleurs, la vitesse est soumise à des variations, car les municipalités françaises ont la possibilité de limiter la célérité des véhicules au sein de leur territoire; ce qui n'est pas le cas au Québec où l'uniformisation de la vitesse demeure.

La principale différence entre les deux territoires réside dans le poids du pouvoir local français dans la législation automobile : dans le Rhône, les acteurs sont plus nombreux à élaborer la normalisation. La normalisation au sein des deux territoires se structure autour de deux textes auxquels s'ajoutent des modifications au fil des ans.

\section{Le Code de la route \\ et la loi concernant les véhicules automobiles : compromis et domination du politique}

En 1921, les différents textes français forment le premier Code de la Route ; au Québec, la loi concernant les véhicules automobiles est sanctionnée le 15 mars 1924 (tableau 3). À la demande des agriculteurs qui se sentent lésés, le Code de la Route est modifié le 31 décembre 1921 et exclu, une fois de plus, ces derniers de la réglementation imposée aux véhicules à moteur. Au Québec, la loi de 1924 accorde aux municipalités la gestion de la circulation et de la vitesse sur leur territoire ; dès lors une pluralité de vitesse est permise.

La vitesse automobile est source d'uniformisation des trajectoires des territoires comme l'indique la normalisation au travers de la réduction de la vitesse, de la nécessité de conditionner les usagers par des connaissances théoriques et pratiques et des prérequis matériels indispensables intégrés au véhicule. Progressivement, la normalisation automobile, dans une optique sécuritaire, circonscrit les véhicules à moteur de telle manière que les conducteurs ont peu de liberté et beaucoup de contraintes.

Tableau 2 La législation automobile dans le Rhône et la Province de Québec (1899-1919)

\begin{tabular}{|c|c|c|c|c|c|}
\hline \multicolumn{3}{|l|}{ Rhône } & \multicolumn{3}{|l|}{ Québec } \\
\hline date & loi & sujet & date & loi & sujet \\
\hline $\begin{array}{c}10 \text { mars } \\
1899\end{array}$ & $\begin{array}{l}\text { décret portant sur } \\
\text { le règlement pour } \\
\text { la circulation des } \\
\text { automobiles }\end{array}$ & $\begin{array}{l}\text { formalités pour la mise en } \\
\text { circulation du véhicule ; } \\
\text { manières de se conduire sur } \\
\text { les routes ; vitesses et } \\
\text { limites de vitesse; } \\
\text { contraventions }\end{array}$ & & & \\
\hline 1901 & & $\begin{array}{l}\text { apposition obligatoire de } \\
\text { plaques d'immatriculation }\end{array}$ & $\begin{array}{r}9 \text { mars } \\
1906\end{array}$ & $\begin{array}{l}\text { loi concernant les } \\
\text { véhicules à } \\
\text { moteur }\end{array}$ & $\begin{array}{l}\text { enregistrement du véhicule } \\
\text { au bureau du trésorier } \\
\text { provincial ; plaques } \\
\text { d'immatriculation ; signal } \\
\text { avertisseur ; lanternes à } \\
\text { l'avant et à l'arrière du } \\
\text { véhicule ; règles de } \\
\text { circulation instaurées }\end{array}$ \\
\hline 1919 & & $\begin{array}{l}\text { apposition d'un signal } \\
\text { sonore et des phares }\end{array}$ & 1909 & statuts refondus & synthèse des précédentes lois \\
\hline
\end{tabular}




\begin{tabular}{|c|c|c|c|c|c|}
\hline \multicolumn{3}{|l|}{ Rhône } & \multicolumn{3}{|l|}{ Québec } \\
\hline date & loi & sujet & date & loi & sujet \\
\hline 1921 & $\begin{array}{l}\text { Code de la } \\
\text { Route }\end{array}$ & $\begin{array}{l}\text { limitation de vitesse ; } \\
\text { identification, éclairage, } \\
\text { dimensions du véhicule ; permis } \\
\text { de conduire ; âgé de } 18 \text { minimum }\end{array}$ & 15 -mars-24 & $\begin{array}{c}\text { loi concernant } \\
\text { les véhicules } \\
\text { automobiles }\end{array}$ & $\begin{array}{l}\text { Synthèse des lois précédentes ; } \\
\text { réglementation de la circulation } \\
\text { accrue ; les municipalités ont la } \\
\text { charge de la réglementation de la } \\
\text { vitesse sur leur territoire }\end{array}$ \\
\hline 31-déc-21 & $\begin{array}{l}\text { Code de la } \\
\text { Route }\end{array}$ & $\begin{array}{l}\text { Agriculteurs ne sont pas } \\
\text { concernés par la majorité des } \\
\text { éléments du Code de la Route }\end{array}$ & & & \\
\hline mai-22 & Décret & $\begin{array}{l}\text { Confirme la singularité des } \\
\text { agriculteurs }\end{array}$ & & & \\
\hline
\end{tabular}

\section{La vitesse bridée : l'accentuation de la normalisation}

Les modifications du Code de la Route et de la loi concernant les véhicules automobiles circonscrivent les véhicules à moteur et plus largement les usagers de la route (tableau 4). Ainsi, en France, le décret du 19 janvier 1933 met fin la domination du cheval en imposant le positionnement du véhicule à droite de la chaussée et non plus au milieu comme c'était le cas auparavant [29]. Au Québec, dès 1925, les municipalités perdent leurs prérogatives relatives à la normalisation automobile et l'uniformisation de la vitesse voit le jour. Après 1945, la normalisation des deux territoires s'oriente vers le renforcement de la sécurité routière, l'essor quantitatif des forces de l'ordre pour veiller à l'application des lois et la sévérité des contraventions en cas de non-respect.

L'élaboration de la normalisation se fait de manière progressive : les textes de loi comblent les manques et les faiblesses des précédents. Conçu comme des compromis entre le politique, les automobilistes, les usagers de la route, ils montrent les interactions entre les différents systèmes de transports. Après 1945, la législation automobile se complexifie au sein des deux territoires $\mathrm{du}$ fait de l'accroissement des usagers et des usages divers de l'automobile. L'automobile, pensée comme un moyen de transport libérateur, enchaîne ses usagers autour de différentes règles plus complexes les unes que les autres.

Tableau 4 L'enchaînement des véhicules à moteur à la normalisation dans le Rhône et au Québec (1932-1960)

\begin{tabular}{|c|c|c|c|c|c|}
\hline \multicolumn{3}{|l|}{ Rhône } & \multicolumn{3}{|l|}{ Québec } \\
\hline date & loi & sujet & date & loi & sujet \\
\hline 05 -oct-32 & décret & $\begin{array}{l}\text { obligation de céder le passage à } \\
\text { droite aux intersections }\end{array}$ & 1925 & $\begin{array}{l}\text { loi concernant les } \\
\text { véhicules } \\
\text { automobiles }\end{array}$ & $\begin{array}{l}\text { pouvoir législatif des } \\
\text { municipalités diminué en matière } \\
\text { d'automobilisme }\end{array}$ \\
\hline 19-janv-33 & décret & $\begin{array}{l}\text { Positionnement du véhicule à } \\
\text { droite sur la chaussée : fin de la } \\
\text { domination du cheval }\end{array}$ & & & \\
\hline Post-1945 & & $\begin{array}{l}\text { refonte du Code de la route : } \\
\text { protéger les usagers et } \\
\text { accroissement de la } \\
\text { normalisation }\end{array}$ & Post-1945 & & $\begin{array}{l}\text { accentuation de la législation et } \\
\text { diminution de la vitesse } \\
\text { automobile }\end{array}$ \\
\hline
\end{tabular}


La culture de la vitesse automobile, graduellement constituée, accentue la capabilité des conducteurs de véhicules motorisés en même temps qu'elle offre un cadre de pensées, de connaissances et de savoir-faire. Néanmoins, les usages et la culture de la vitesse automobile ne sont rien sans une infrastructure cohérente et entretenue qui sous-tend la mobilité.

\section{L'automobiliste qui désire être mobile requiert une infrastructure routière viable}

N'importe quel mode de transport n'est rapide que par le degré d'efficacité et d'efficience de l'infrastructure qui l'héberge [30]. L'automobile n'échappe pas à la règle : un réseau routier est progressivement élaboré permettant aux conducteurs de circuler aisément, de façon sécuritaire et rapidement.

\section{De la terre à l'asphalte : adapter l'infrastructure à l'automobile}

En 1919, on assiste à une asymétrie de la situation de départ dans le Rhône et dans la région de Québec concernant le réseau routier qui finit par s'équilibrer en 1961.

Dans le département du Rhône, le maillage routier est dense bien qu'inadapté aux véhicules à moteur [31]. Le service des Ponts et chaussées hiérarchise et restructure le réseau routier autour de Lyon afin de l'optimiser ; cela passe par l'échec du projet autoroutier Lyon-Saint-Étienne en 1935. Les associations telles que l'ACR, le TCF (TouringClub de France), bien avant 1919, font pression auprès des instances publiques et subventionnent la construction de routes. Après la Seconde Guerre mondiale, le réseau routier du Rhône est reconstruit et amélioré. Dès les années 1950, des plans quinquennaux sont appliqués qui témoignent de la mainmise des pouvoirs publics sur la voirie. La nouvelle tentative de projet autoroutier durant les années 1950 annonce les prémisses d'un nouveau système : «l'ère de la grande vitesse » [16].

En ce qui concerne la région de Québec, le réseau routier est peu étendu et en piteux état du fait de la préférence pour le transport ferroviaire et maritime [32]. Les requêtes pour obtenir un réseau routier adéquat pour les véhicules à moteur se multiplient de la part des instances locales, des politiciens ainsi que du CAQ. Le ministère de la Voirie - fondé en 1914 - élabore une politique visant à densifier le maillage routier, à hiérarchiser et à améliorer l'état des routes; la tentative, à l'aube des années 1930, d'établir un réseau autoroutier indique que l'ère de la grande vitesse est prématurée. Pour remédier aux rudesses du climat hivernal, le ministère de la Voirie, sous l'impulsion du CAQ, inaugure l'entretien de chemins d'hiver dès 1928 permettant ainsi aux véhicules à moteur de circuler tout au long de l'année - et non plus six mois. Cette initiative est suivie par les municipalités et les entrepreneurs privés. À la fin des années 1950, la plupart des routes principales sont ouvertes à la circulation automobile [33]. Après 1945, l'État québécois accroît sa mainmise sur le réseau routier avec la constitution de trois ministères : le ministère de la Voirie qui construit et entretient les routes, le ministère des Travaux Publics qui construit les ponts et le ministère des Transports qui régit la circulation et la sécurité routière. Il inaugure dès 1955 un plan quinquennal pour améliorer le réseau routier, le budget de la voirie augmente et le réseau routier se densifie. Dès l'aube de la décennie 1950, des institutions sont constituées pour établir la route la plus longue du Canada - la Transcanadienne.

Durant la première moitié $\mathrm{du} \mathrm{XX}^{\mathrm{e}}$ siècle, le chemin poussiéreux se transforme progressivement. Dans le Rhône, le réseau routier, bien maillé du fait d'héritages historiques, requiert un revêtement adéquat alors que dans la région de Québec, l'automobile impulse la modification des chemins en routes. Les associations automobiles - l'ACR et le CAQ -, institués en groupe de pression, effectuent quelques travaux mineurs destinés à mobiliser les pouvoirs publics [34]. Ces derniers donnent au réseau routier sa hiérarchisation et son organisation se structure entre les diverses échelles administratives - l'État ou le ministère de la Voirie - pour le Québec -, le département - pour le Rhône - , la commune ou la municipalité.

Les mutations entourant le réseau routier, désormais structuré, entretenu et viable, sont plus profondes : le paysage est remanié aux abords des routes.

\section{Faire un pas de côté : l'attention indispensable aux road-sides, facteurs de la vitesse automobile}

Les abords de la route sont aménagés pour améliorer la circulation par l'essor d'une signalisation routière à destination des conducteurs de véhicules à moteur et par les remaniements relatifs à la flore environnant la route.

\section{Signer la terre : signaliser la route}

Dans le département du Rhône, trois institutions s'activent dans la pose et l'entretien de panneaux de signalisation : le service de voirie, l'ACR et le TCF. À partir de 1919, les principes généraux de la signalisation de direction sont officialisés par décret en France. La signalisation routière se multiplie et se diversifie notamment à proximité des carrefours. Du fait de la célérité des véhicules à moteur, les institutions chargées de la signalisation routière se tournent vers la symbolisation des indications plutôt que 
les panneaux textuels. Ce faisant, elle entre en concurrence avec les autres panneaux - commerciaux, publicitaires, politiques ou culturels. La conférence de Genève de 1931 uniformise la signalisation à l'échelle européenne autour de cinq catégories : les signaux de danger, les signaux de prescription absolue, les signaux d'obligation à exécuter, les signaux de prudence et les signaux d'indication. Au cours des années 1940 et 1950, la signalisation routière est réorganisée sous le coup de plusieurs décrets ; quatre catégories de panneaux sont identifiables : les signaux de danger sous forme triangulaire; les signaux de prescriptions absolues sous forme circulaire ; les signaux d'indication sous forme rectangulaire - signaux de localisation, de direction, signaux touristiques ou d'intérêt local et signaux de chantier. Parallèlement, la loi du 12 avril 1943 réglemente l'affichage publicitaire aux abords de la route. Bien que contingenté dans une position de groupe de pression, l'ACR, par l'intermédiaire de la FNAC (Fédération nationale des automobiles-clubs de France), s'implique tout de même dans la présignalisation, soit le fait de positionner des panneaux 200 mètres avant la situation ; par ailleurs, il finance l'achat de panneaux et engage des démarches auprès des pouvoirs publics pour que les panneaux d'interdiction apparaissent sous fond rouge et les panneaux de direction et de permission sous fond bleu.

Au sein de la région de Québec, le CAQ encourage le ministère de la Voirie en posant des panneaux entre 1921 et 1924 - panneaux de danger, des indications routières, des signaux à proximité des hôpitaux et des écoles. Dès 1922-1923, le ministère de la Voirie installe des panneaux indicateurs de vitesse et des signaux routiers. Sous la responsabilité d'un assistant-ingénieur, J.-Omer Martineau, les panneaux textuels sont remplacés par des panneaux symboliques. À cela s'ajoutent les panneaux bilingues installés par la Commission des monuments historiques, dès 1922, qui ont pour but de valoriser les richesses de la province - architecturales, naturelles, etc. -, la numérotation des routes et dès 1929 le panneau géographique signalant les lieux naturels tels que les rivières, les montagnes et les îles. À l'aube des années 1930, on dénombre une trentaine de panneaux routiers qui avertissent, orientent et informent les usagers de la route. Cette multiplication des indications routières rentre en concurrence avec les enseignes publicitaires et commerciales ; si bien que dès 1924 et 1927 , le ministère de la Voirie interdit la pose d'affiches par des individus et des institutions privées telle que le CAQ. Ce dernier se tourne donc vers la rénovation des croix aux abords de la route et mobilise les pouvoirs publics pour obtenir plus de signalisation routière. L'accroissement de l'anglophonie et de l'américanisation constitue une autre tension au sein de la signalisation routière au début des années 1930 : les revues les plus nationalistes du Québec n'hésitent pas à parler de « route désenchantée » [35]. Les différents entrepreneurs cherchent à faire du profit en affichant leurs produits, tandis que l'État joue le rôle de régulateur. Pour enrayer ce phénomène, des concours annuels d'enseignes organisés par le ministère de la Voirie voient le jour. Mandaté par les pouvoirs publics, l'abbé Albert Tessier, dans son Rapport sur le tourisme de 1939, critique ostensiblement les affiches anglophones des bords des routes. Le ministère de la Voirie s'attache alors à faire retirer les affiches commerciales anglophones vantant le Coca-Cola, le Pepsi-Cola, les hot-dogs, les Chicken Dinners, etc. [36]. À partir de 1949, est créé au sein du ministère de la Voirie un service chargé de la réglementation des affiches le long des routes. Dans le même temps et durant les années 1950, les motels apparaissent et valorisent leur commerce par les affiches : ils font redoubler le spectre de l'américanisation. En 1949, la conférence sous l'égide des Nations Unies traitant du transport routier et automobile avalise une signalisation spécifique au continent nord-américain ; malgré le fait que la province de Québec conserve le bilinguisme de sa signalisation - par exemple, le panneau « Arrêtez-Stop ».

La signalisation routière, encouragée par les associations automobiles, est reprise par les pouvoirs publics qui y imposent leur mainmise. Celle-ci, de plus en plus complexe, amène à une rationalisation du rapport au territoire par les individus et une scientificité du pouvoir politique - il devient un expert. Le cas québécois est spécifique puisque, dès années 1930 jusqu'à la fin de la période étudiée, l'automobiliste américain fait surgir la problématique de l'anglophonie des enseignes qui mettent en danger la culture et son identité québécoise.

Signalé, le bord de route est aussi réaménagé de manière esthétique.

\section{La route herborisée}

La construction et l'aménagement des routes pour la vitesse automobile amènent à une modification de la flore. Cette thématique est traitée au sein des deux territoires, mais de manière différente.

Dans le Rhône, la plantation des arbres en bordure de route remonte au XVIII ${ }^{\mathrm{e}}$ siècle [37]. L'irruption de l'automobilisation amène à une redéfinition de la législation entre responsabilité publique et responsabilité privée. Pour les chemins ruraux, le maire est responsable de « l'élagage ou [de] la réduction des haies d'une certaine hauteur. En ce qui concerne les chemins vicinaux, le préfet a le même pouvoir [...] » [38]. Par contre, concernant les routes départementales et nationales, la législation est muette. Se pose fondamentalement la question de l'utilité ou non des 
arbres aux abords des routes [39]. D'un côté, ils sont la source de dangers potentiels pour les véhicules qui font une sortie de route. D'un autre côté, il en va du caractère esthétique du paysage et de la fonction utilitariste des arbres comme protection de la route et de l'usager contre les conditions climatiques - ensoleillement, pluie, grêle et autres facteurs climatiques. Par ordre du préfet, les haies les plus proches de la voie de circulation ne doivent pas dépasser un mètre de hauteur, sous peine de les faire enlever aux frais de l'intéressé [40]. Cette nouvelle mesure est donc accueillie par des contestations de la part des riverains [40]; contestations qui se poursuivent tout au long $\mathrm{du} \mathrm{XX}^{\mathrm{e}}$ siècle [41]. Toutefois, la question des arbres est rapidement délaissée du fait de son caractère historique et de l'essor de l'éclairage des routes.

Dans la région de Québec, l'embellissement de la route est pris très au sérieux par le ministère de la Voirie, car il est un des facteurs d'accroissement économique via le tourisme [42]. Il entreprend donc, sur les routes principales, puis sur les routes secondaires, la plantation de variété d'arbres supportant relativement les conditions climatiques et ne ralentissant pas l'entretien des chemins d'hiver - érables, chênes, noyers, hêtres, tilleuls, pins [43]. Toutefois, un nombre d'individus s'y oppose en les dégradant ou bien en les déracinant, alors que d'autres se contentent de déraciner les arbres pour les replanter dans leur propriété [44]. Pour remédier à cette situation, le gouvernement place ceux-ci sous la Loi de protection des arbres en 1928-1929 [45]. Le mouvement de plantation s'essouffle au tournant des années 1930 ; de plus, les rapports du ministère de la Voirie de Québec ne font plus mention de la flore après 1933.

L'esthétisation du bord de route par la plantation d'arbres est principalement le fruit des pouvoirs publics, plus ou moins soutenu par les clubs automobiles. Ce sujet constitue une véritable thématique dans la société québécoise participant de la mise en tourisme du territoire, alors que dans le Rhône le caractère historique le présente comme moins visible.

Le paysage devient structuré par la signalisation routière et par la présence des arbres. L'espace entourant la route devient progressivement hiérarchisé et soumis à une législation stricte. Cela se présente dans un double mouvement cohérent : d'un côté, un accroissement des connaissances et des savoir-faire en lien avec l'espace routier et de l'autre une mise à distance de l'environnement par la scientifisation du paysage de la vitesse. L'absence ou la présence d'arbres permet de rythmer les déplacements des individus sur la route : cela chasse la monotonie du voyage et permet la vigilance de l'usager. La modification des bords de route passe aussi par la constitution des infrastructures liées à l'économie automobile : les garages et les stations-service.

\section{Les structurations des road-side}

\section{De non-lieux aux infrastructures économiques : les garages automobiles}

Le garage constitue une institution dans les mondes de l'automobile. S'il est plutôt aisé de trouver un garage en milieu urbain, ce n'est pas le cas dans l'espace rural. En l'absence de garage, les professionnels du fer tels que le maréchal-ferrant, le forgeron, le charron et le ferblantier sont amenés à aider les automobilistes. La question de la définition du garage pose donc problème.

En 1920, un département fortement automobilisé comme le Rhône ne présente aucun garage, excepté à Villefranchesur-Saône. Cette absence surprend, mais elle peut être imputée au caractère élitiste de l'automobile, au savoir-faire des chauffeurs, des automobilistes et des artisans ruraux capables de dépanner les conducteurs. Moins de vingt ans plus tard, la situation est bien différente : on dénombre presque 160 garages en 1939. Ces derniers se localisent principalement dans la vallée du Rhône : depuis Belleville-sur-Saône en passant par Villefranche-sur-Saône et Lyon et en allant jusqu'à Condrieu. Principalement installés sur la rive ouest du Rhône, ils se retrouvent le long des routes nationales - nationale 6 et nationale 86 . Ailleurs, des garages se localisent sur d'autres nationales - nationale 89 et nationale 7 - en direction de St-Étienne et du département de la Loire. La route nationale, par le trafic qu'elle draine, incite à l'implantation des garages. Le reste du département est dépourvu de garage, soit l'est de Lyon, les régions montagneuses - les monts du Lyonnais du Beaujolais - et le nord du département ; espaces peu peuplés, difficiles d'accès et mal reliés avec les différentes routes nationales qui traversent le département (carte 1).

En 1959, le nombre de garages a augmenté de $142 \%$ - soit 227 garages (carte 2). Ils se sont multipliés dans les communes où ils étaient déjà présents en 1939 du fait de la hausse de la demande des usagers. C'est le cas de Villefranche-sur-Saône - de 18 en 1939 à 23 garages en 1959 -, de Caluire-et-Cuire de sept à dix. Le garage comme infrastructure consolide sa position aux abords des routes. Quelques communes telles Thizy et Amplepuis voient une diminution du nombre de garages : cela montre la compétitivité qu'il existe dans ce domaine. En l'espace d'un demi-siècle, le garage automobile est désormais reconnu comme tel par les usagers, les pouvoirs publics et la société.

Outre-Atlantique, on retrouve ce flou entourant la définition du garage et l'appel aux métiers du cheval pour résoudre les problèmes mécaniques durant les années 1920. Au tournant des années 1930, les garages se multiplient prenant le pas sur les ateliers de forge [46]. La première définition 


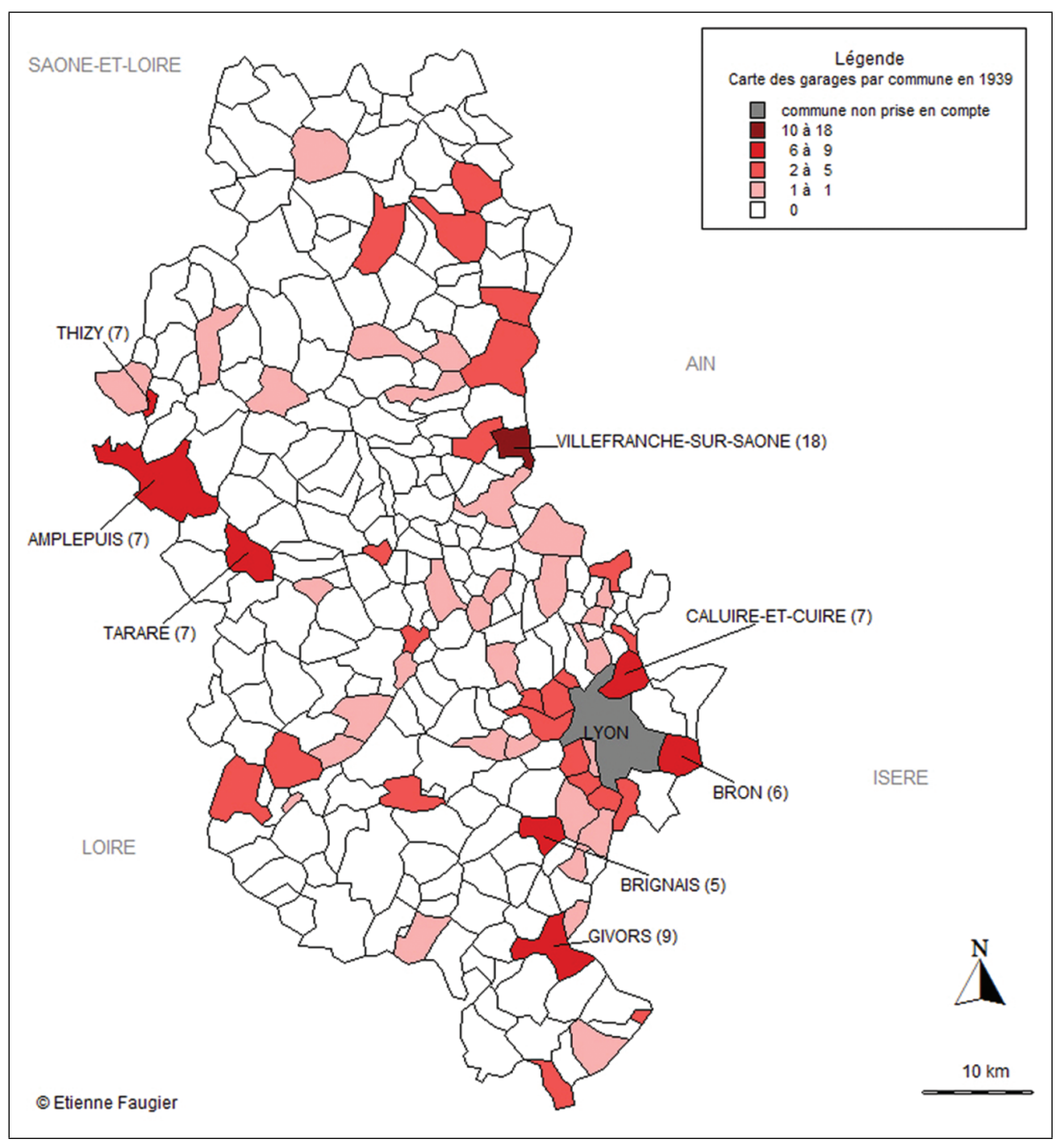

Fig. 1 Localisation des garages par commune dans le département du Rhône en 1939

du garage date de 1931 et décrit cette infrastructure comme un établissement vendant des pneus et des accessoires et réparant les véhicules automobiles. Dans la région rurale de Québec, le nombre des garages et réparations d'autos augmente jusqu'en 1948 - 78 garages au total - puis diminue en 1956 - 60 garages - pour se stabiliser après 1960 - 67 garages [4]. La majorité des garages se localisent, sur la Côte-de-Portneuf et la Côte-de-Beaupré, à proximité du Saint-Laurent sur la route de grande communication menant de Trois-Rivières à La Malbaie en passant par Québec (carte 3). Le comté de Portneuf domine la région rurale de Québec en termes d'infrastructures automobiles; on peut avancer l'hypothèse qu'il s'agit d'un territoire industrialisé. Le faible nombre d'infrastructures dans le comté rural de Québec s'explique par la concentration de ces dernières dans le pôle urbain de Québec. L'île d'Orléans accueille peu de garages puisque celle-ci est plutôt isolée et est soumise à des réglementations strictes concernant l'implantation de commerces et de structures de ce genre.

Pour les deux espaces, les garages émergent progressivement comme une institution à part entière. Ils atteignent leur pleine maturité à la fin des années 1950. Certains individus sont contraints d'abandonner l'économie du cheval pour celle de l'automobilisme, tandis que d'autres sont séduits par ce dernier : la conquête de l'automobilisme atteint donc la sphère économique par le biais des individus. Les garages se localisent principalement sur les grandes artères de circulation - dans le Rhône, les routes nationales ; dans la région de Québec, la route de grande communication Trois-Rivières-La Malbaie. Cette infrastructure constitue un élément principal soutenant le système de la vitesse automobile. 


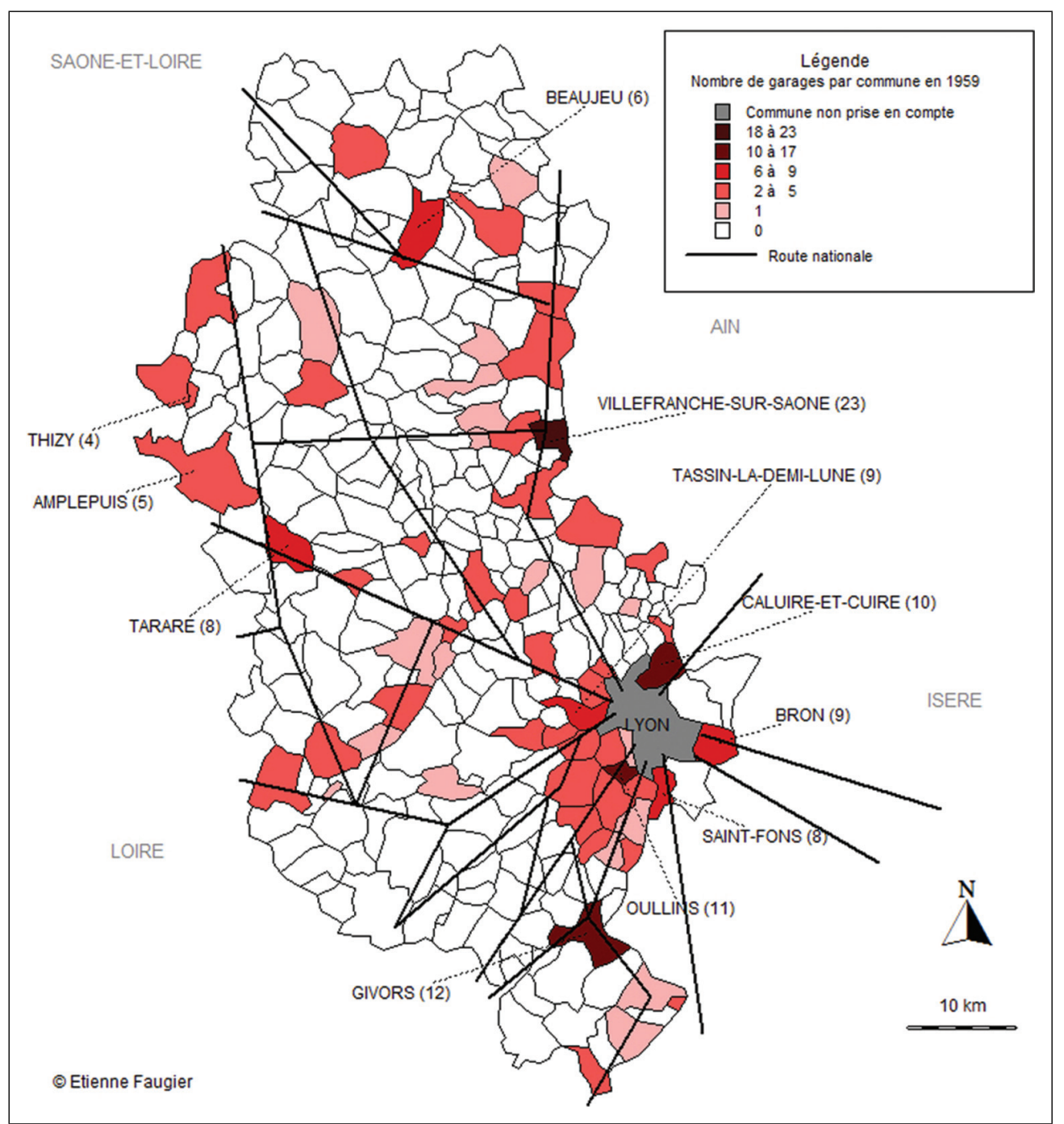

Fig. 2 Localisation des garages sur les routes nationales du Rhône en 1959

\section{Matérialiser le carburant : des bidons d'essence aux stations-essence}

Au sein des deux territoires, l'approvisionnement des automobiles en essence se fait en recourant aux bidons d'essence durant les deux premières décennies du $\mathrm{XX}^{\mathrm{e}}$ siècle environ. Ils se trouvent chez divers commerçants comme l'épicier, le forgeron, le pharmacien et les professions travaillant le fer. Dès les années 1920, les pompes à essence font leur apparition de part et d'autre de l'Atlantique [46, 47].

Dans le département du Rhône, à la fin des années 1950, la localisation géographique des pompes à essence répond à diverses logiques (carte 4). Tout d'abord, la majorité d'entre elles se localisent autour du centre urbain majeur qu'est Lyon et sur l'axe routier principal Villefranche-sur-SaôneLyon-Givors. Ensuite, celles présentes dans les monts du Lyonnais et du Beaujolais restent exceptionnelles et sont localisées le long des axes routiers. De manière générale, le nombre minime de postes d'essence s'explique par leur présence au sein d'autres commerces comme l'épicerie du village, l'atelier du maréchal ferrant ou d'autres [48]. La majorité d'entre elles, en 1956, appartiennent aux grandes entreprises pétrolières : Shell, Antar, Esso, Mobil Oil française, Excelsior, société Huiles \& Combustibles Liquides, société française des Combustibles Liquides. Les autres postes d'essence ou pompes à essence appartiennent à ces entreprises qui ont passé un accord avec le commerçant. Dès lors, l'institutionnalisation des entreprises pétrolières voit le jour à travers cette infrastructure : elles se rendent visibles par ces relais automobiles.

Dans la région de Québec, les stations d'essence s'organisent en trois catégories. La première vend principalement de l'huile et de l'essence ; la seconde inclut, en plus de la vente de carburant, des pièces et des accessoires automobiles ; enfin, la dernière étend son commerce à la vente de boissons et d'aliments - crème 


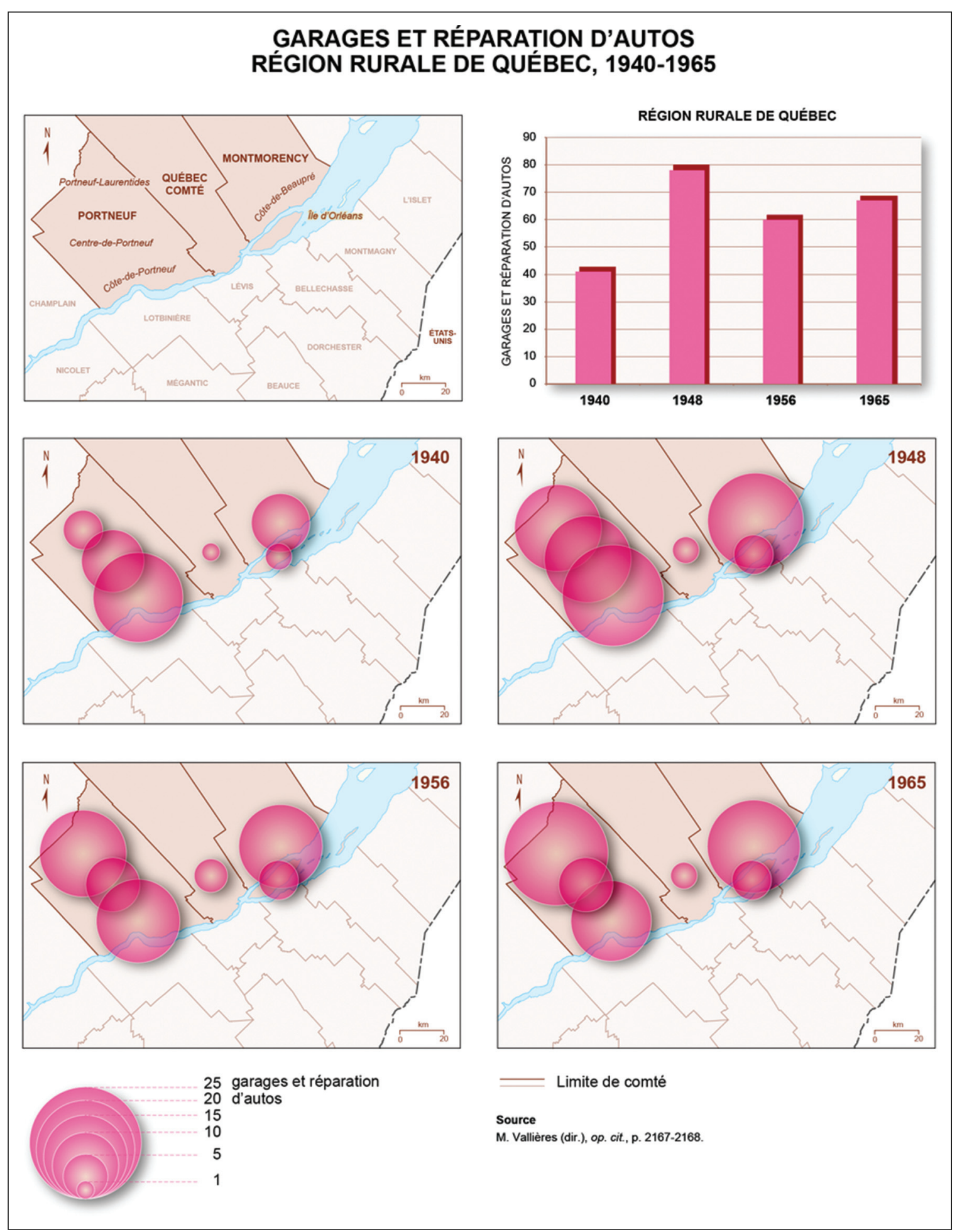

Fig. 3 Répartition des garages et réparation d'autos dans la région rurale de Québec (1940-1965)

glacée, bonbons, tabac et autres. Celles-ci s'approprient une part du commerce de la route en diversifiant leurs produits : produits destinés au véhicule à moteur et produits pour l'usager. En 1940, il n'y a aucun détaillant d'essence sur la rive nord de la région de Québec (carte 5). Moins de dix ans plus tard, ils sont localisés sur l'artère centrale de la rive nord - le chemin du Roy - et dans le comté de Portneuf, principalement la Côte-de-Portneuf. Entre 1946 


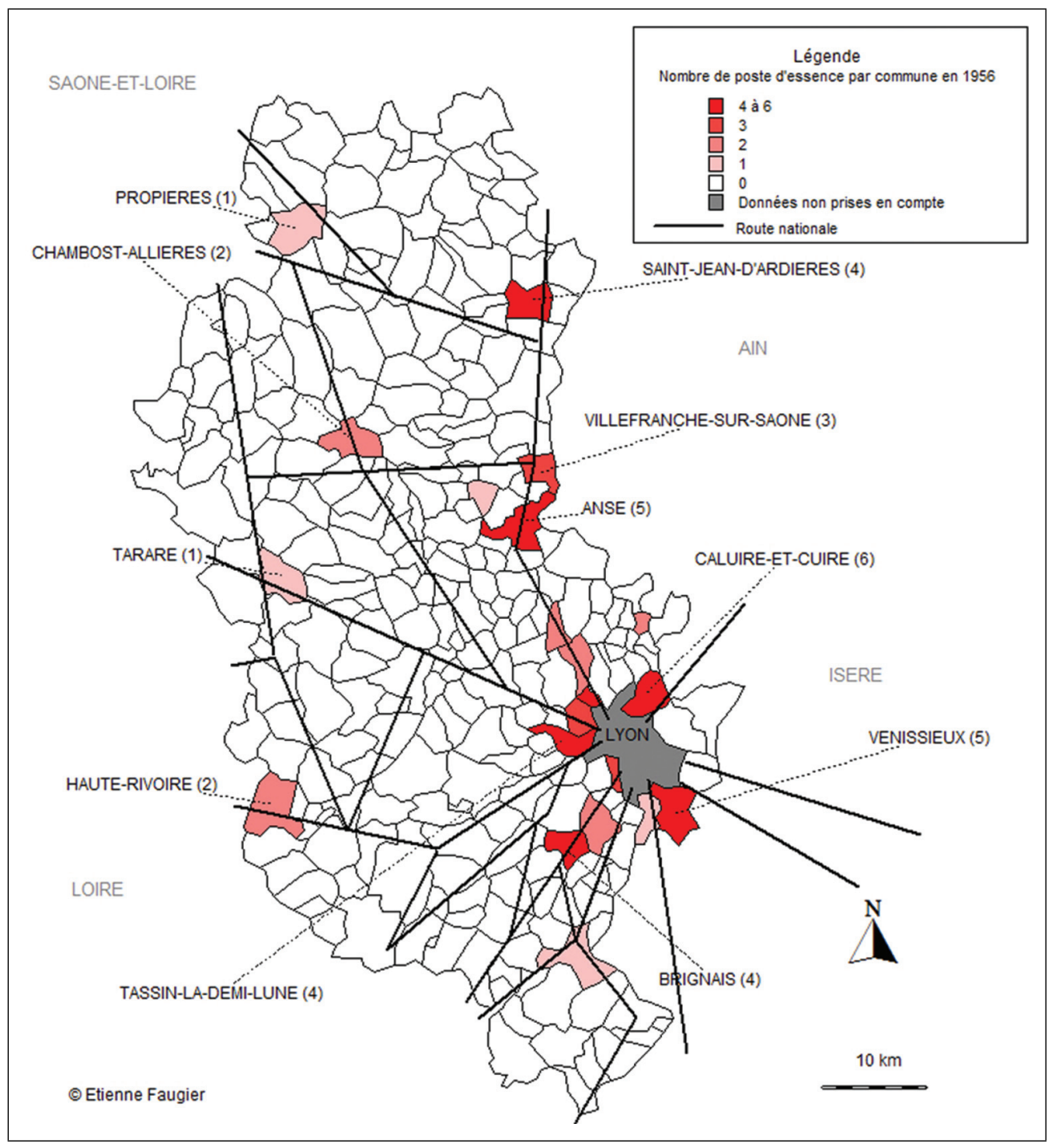

Fig. 4 Nombre de postes d'essence par commune dans le département du Rhône en 1956

et 1958 , le nombre de détaillants augmente fortement de sept à 58 ; ils viennent consolider le réseau routier de la rive nord en formant un cordon d'approvisionnement pour les usagers automobiles. Les détaillants d'essence augmentent en nombre parallèlement à la hausse des conducteurs dans l'après Seconde Guerre mondiale. Par le choix de leur situation géographique, ils soulignent la structuration du territoire tout en appuyant celle-ci.

Les garages et les stations-service se multiplient aux abords des grandes artères du réseau routier. D'une certaine manière, elles viennent supplanter les commerces dédiés à l'économie chevaline. Cela témoigne du parti pris de la population pour le système de la vitesse automobile. Ces infrastructures de bord de route, souvent ignorées ou négligées par les chercheurs, constituent le cordon vital du système de la vitesse automobile. Ces établissements qui peuvent être assimilés à des «non-lieux » [49] n'en constituent pas moins des points de contact indispensables aux usagers motorisés de la route.

Entre 1919 et 1961, des modifications aussi profondes que diverses sont introduites dans l'environnement des deux territoires par le biais de l'automobilisation. Celles-ci entraînent l'élaboration plus prononcée d'un paysage de la vitesse liant l'automobile au réseau routier [16]. Ce paysage, d'abord ébauché par les associations automobilistes, est imposé puis fondu dans l'environnement par l'administration publique. Les deux territoires étudiés empruntent des chemins sensiblement similaires avec les travaux de voirie, la pose de panneaux et la plantation d'arbres au bord des routes. La région de Québec fait face à l'élaboration d'un réseau routier par de massifs investissements financiers et surmonte, avec divers acteurs, le climat hivernal rigoureux par l'entretien des chemins d'hiver. La patrimonialisation de la flore n'intervient qu'après 1960 dans le Rhône, alors 


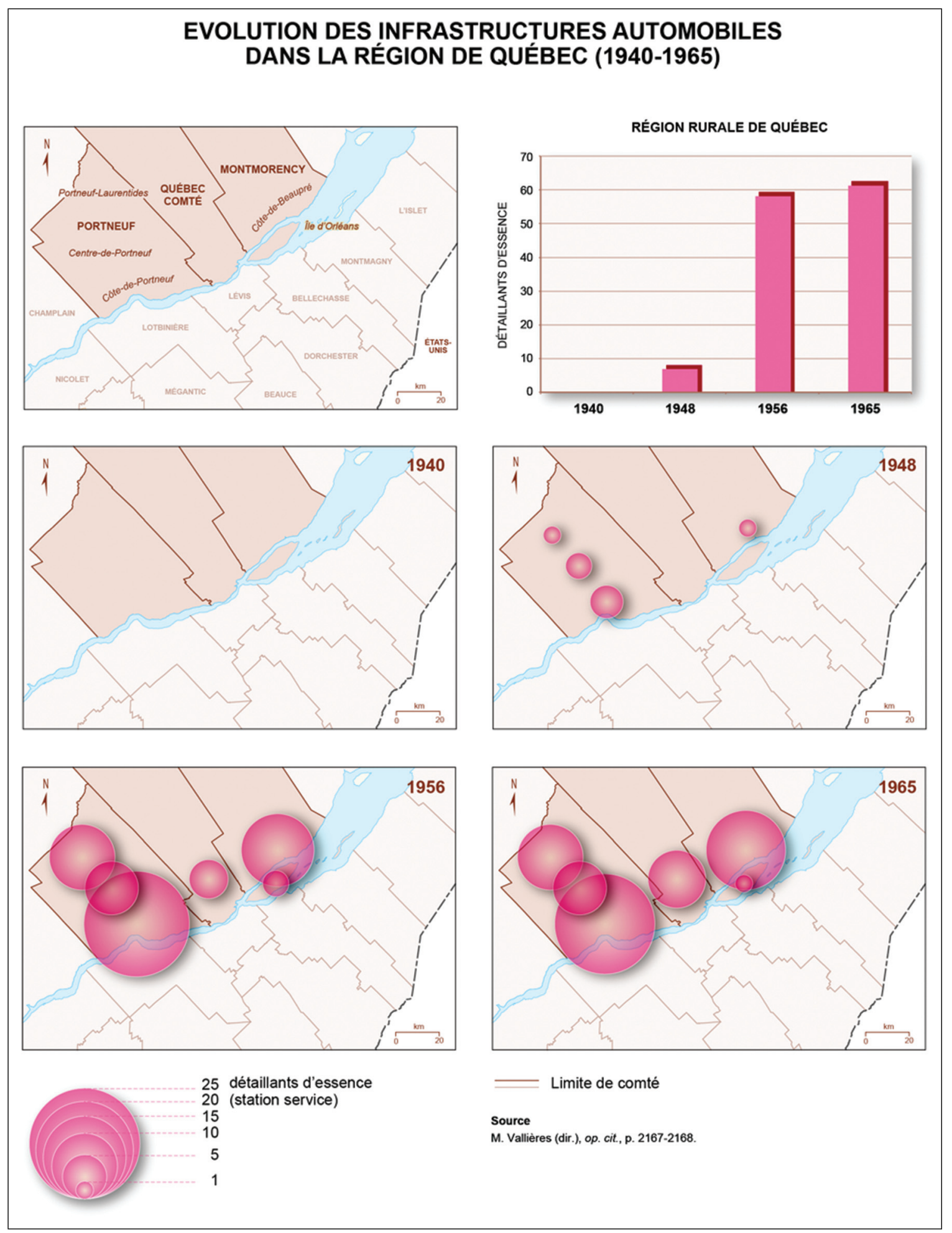

Fig. 5 Répartition des détaillants d'essence par comté dans la région de Québec (1940-1965)

que dans la région de Québec, des mesures patrimoniales sont rapidement imposées pour protéger l'environnement et la nature. La route, enjeu économique, magnifié par l'automobile est devenue un enjeu politique, social et culturel. La signalisation et l'embellissement des routes participent d'une esthétique de la vitesse : la recherche de beauté de la part des administrations se matérialise sur les routes pour former un espace cohérent et logique. 
La restructuration de celles-ci, leur entretien, l'élaboration d'une signalisation routière, l'embellissement des bords de route et l'essor d'infrastructures économiques permettent à l'individu, par l'intermédiaire des véhicules à moteur, de faire usage de la célérité dans ses déplacements.

\section{La vitesse : quels apports de la perspective historique?}

La vitesse automobile séduit les populations, qu'il s'agisse des pionniers, des associations automobiles ou encore des professionnels de l'économie automobile. Ils constituent les premiers promoteurs de la vitesse automobile au travers des usages qu'ils développent, de la culture qu'ils promeuvent et du réseau routier qu'ils appellent de toute leur voix. Les actions qu'ils ont engagées sont reprises et amplifiées par les pouvoirs publics à l'échelle locale comme à l'échelle nationale ou fédérale. La vitesse automobile effectue ainsi une véritable révolution au sein des acteurs de la société : ils sont acquis progressivement à sa cause.

À travers la constitution et les usages de la vitesse automobile, le croisement des deux territoires met l'accent sur leur uniformisation progressive en les insérant dans la même trajectoire et une chronologie qui se recoupe après 1945. Cela tient pour partie de la mobilisation d'acteurs similaires : les associations automobiles, les pouvoirs publics, les usagers qui ont des besoins et des mobilités relativement similaires. On observe une homogénéisation de l'offre et de la demande automobile, des mutations environnementales, politiques et culturelles au sein des deux sociétés étudiées. Il y a donc une "polygénèse écologique » entre les deux territoires [50].

La vitesse - automobile - s'insère au sein même des mobilités : elle est à la fois sa source et son expression. Elle prend différentes formes : les déplacements concrets, les infrastructures économiques, le réseau routier, les réaménagements des bords de route, les littératures et la normalisation.

Les mobilités font le système de transport et le système de transport fait les mobilités. Les usagers de l'automobilisme, par leur mobilité, appellent à la constitution du système de la vitesse automobile auquel le politique tente de répondre.

L'approche historique de la vitesse s'avère relativement utile pour appréhender l'époque contemporaine. Ainsi, la progressive remise en cause de l'automobile ou de l'infrastructure routière entraîne irrévocablement le questionnement de notre propre culture, chose que les contemporains ont tendance à négliger. L'adoption de la vitesse automobile par les sociétés occidentales les conduit vers une configuration similaire; ce faisant, les problèmes et les limites rencontrés par certains pays devraient intéresser les autres puisqu'ils se trouveraient potentiellement, dans un futur proche, dans la même situation : cela devrait donc encourager les nations à plus de dialogue les unes avec les autres.

Dans cet article, nous avons mis l'accent sur la vitesse autour de l'objet technique qu'est le véhicule à moteur individuel. Cependant, le phénomène peut être étendu aux autres véhicules motorisés tels que l'autobus ou le tracteur pour ce qui est du monde rural [5]. Si notre article présente une vision historique, une vision plus contemporaine des nouveaux usages peut être envisagée en mobilisant les concepts de « vitesse différenciée » [12] et « d'accélération » [51] afin de mettre en évidence l'usage pluriel des systèmes de transport par les individus.

\section{Références}

1. Roche D (2006) Les mobilités concrètes, $\mathrm{XVI}^{\mathrm{e}}-\mathrm{XX}^{\mathrm{e}}$ siècle. French Historical Studies 29(3): 513-515.

2. Schivelbusch W (1990) Histoire des voyages en train. Le Promeneur, Paris.

3. Flonneau M (2008) Les cultures du volant: essai sur les monders de l'automobilisure $X V^{\mathrm{e}}-X X l^{\mathrm{e}}$ siècles, Autrement, Paris.

4. Vallières M (2008) (dir) Histoire de Québec et de sa région. Presses de 1'Université Laval.

5. Faugier E (2013) L'économie de la vitesse : l'automobilisme et ses enjeux dans le département du Rhône et la région de Québec 1919-1961. Thèse de doctorat d'histoire en cotutelle, Université Laval/Université Lumière Lyon 2.

6. Vigour C. (2005) La comparaison dans les sciences sociales : pratiques et méthodes. La Découverte, Paris.

7. Lüsebrink H-J (2003) Transferts culturels transatlantiques et circulation des savoirs dans les cultures populaires - le cas des almanachs de Benjamin Franklin. Tangence 72 : 27-40.

8. Douki C, Minard P (2007) Histoire globale, histories connectées : un changement d'échelle historiographique ? Revue d'histoire moderne et contemporaine 5, $\mathrm{n}^{\circ}$ 54-46: 7-21.

9. Werner M, Zimmermann B (dir.) (2004) De la comparaison à l'histoire croisée. Ed. du Seuil, Paris.

10. Kott S, Nadau T (1994) Pour une pratique de l'histoire sociale comparative, Genèses 17 : 103-111; Ross K (1997) Rouler plus vite, laver plus blanc : la culture française au tournant des années soixante. Abbéville, Paris.

11. Duffy E (2009) The Speed Handbook: Velocity, Pleasure, Modernism. Duke University Press, Durham \& London; Pichois C (1973) Littérature et progrès : vitesse et vision du monde. Éditions de la Baconnière, Neuchâtel.

12. Ollivro J (2006) Quand la vitesse change le monde : essor de la vitesse et transformations des sociétés. Éditions Apogée, Rennes; Ollivro J (2000) L'homme à toutes vitesses : de la lenteur homogène à la rapidité différenciée. Presses Universitaires de Rennes, Rennes; Virilio P (1977) Vitesse et politique : essai de dromologie. Éditions Galilée, Paris; Wiel M (2006) Éloigner les méfaits de la vitesse, Excès de vitesse $5: 10-13$.

13. Virilio P (1995) La vitesse de libération : essai. Éditions Galilée, Paris; Rousseau P (1963) Histoire de la vitesse. PUF, « Que saisje ? », Paris.

14. Studeny C (1990) Le vertige de la vitesse : l'accélération de la France : 1830-1940. Thèse de doctorat d'histoire, Paris, EHESS. 
15. Guigueno V (2010) «Vitesse » in Delporte Christian, Mollier Jean-Yves, Sirinelli Jean-François (dir.), Dictionnaire d'histoire culturelle de la France contemporaine. PUF, Paris: 849-851.

16. Guigueno V (2009) Les paysages de la vitesse in Mathieu Flonneau \& Vincent Guigueno (dir.), De l'histoire des transports à l'histoire de la mobilité ?, PUR, Rennes : 97-102; Desportes M (2005) Paysages en mouvement : Perception de l'espace et transports $\left(X V I I I^{\mathrm{e}}-\mathrm{X} \mathrm{X}^{\mathrm{e}}\right.$ siècle). Gallimard, Paris.

17. Mauch C, Zeller T (2008) The World Beyond the Windshield: Roads and Landscapes in the United States and Europe. Ohio University Press, Athens, Ohio \& Stuttgart.

18. Caron F (1998) La naissance d'un système technique à grande échelle : le chemin de fer en France (1832-1870). Annales HSS 4-5 : 859-885; Edgerton D (1998) De l'innovation aux usages : dix thèses éclectiques sur l'histoire des techniques. Annales HSS 4-5 : 815-837; Hughes TP (1998) L'histoire comme systèmes en evolution. Annales HSS 4-5 : 839-857.

19. Dumont P (1965) Au temps des automobilistes. Edita Lazarus, Lausanne: 148 .

20. Marinetti FT (1909) Manifeste du Futurisme, Le Figaro, 20 février.

21. Bishop CW (1971) La France et l'automobile. Contribution française au développement économique et technique de l'automobile des origines à la deuxième guerre mondiale. Paris, Éd. M.-Th. Génin; Loubet J-L (2001) Histoire de l'automobile française, Paris, Seuil.

22. Gardon S (2009) Gouverner la circulation urbaine : des villes françaises face à l'automobile (années dix - années soixante). thèse de doctorat de science politique, Lyon, Université Lumière Lyon 2.

23. Desrosiers G (2003) Mémoires d'un médecin de campagne: pionnier des bibliothèques municipales-rurales du Québec. Des Rosiers et associés, Montréal: 123.

24. Kline R, Pinch T (1996) Users as Agents of Technological Change: The Social Construction of the Automobile in the Rural United States. Technology and Culture 37(4): 763-795.

25. Fanica P-O (2008) Le lait, la vache et le citadin : du XVII au $X X^{\mathrm{e}}$ siècle. Éditions Quae, Paris.

26. Lecouturier Y (2009) La création du service automobile des PTT en 1946. Les Cahiers de la FNARH 112: 75-80.

27. Fédération des Syndicats d'initiative de la vallée du Rhône (1928) Vallée du Rhône, union des Fédérations des Syndicats d'initiative.

28. Gagnon S (2003) L'échiquier québécois du tourisme. PUQ, Montréal.

29. Orselli J (2009) Usages et usagers de la route : pour une histoire de moyenne durée (1860-2008). Thèse de doctorat d'histoire, Paris, Université Paris 1 Panthéon-Sorbonne: 71.
30. Latour B (2009) Les moteurs immobiles de la mobilité in Flonneau Mathieu \& Guigueno Vincent (dir.), De l'histoire des transports à l'histoire de la mobilité ?, PUR, Rennes : 7-9.

31. Reverdy G (2007) Les routes de France du XX $X^{\mathrm{e}}$ siècle (1900-1951). ENPC, Paris.

32. Blanchard R (1960) Le Canada Français : province de Québec, étude géographique. Arthème Fayard, Montréal.

33. Paquet S (2001) Historique des transports et des déplacements au Québec. Essai en aménagement du territoire et développement régional », Québec, Université Laval.

34. Faugier E (2014) À la croisée des chemins: le Club Automobile de Québec comme cheville ouvrière de l'automobilisme (1912-1948) in Boivin Jérôme \& Savard Stéphane (dir.), Les groupes de pression au Québec. Septentrion, Québec. 74-99.

35. (1949) La route désenchantée. L'Action nationale 34(2) : 81-87.

36. Tessier A (1939) Rapport sur le tourisme. n.c.

37. Bonnevay L (1946) Histoire politique et administrative du Conseil général du Rhône 1790-1940. tome 2, Éd. Joannès Desvigne, Lyon: 202.

38. ACR (Automobile-Club du Rhône) (1928) L'élagage des arbres et des haies aux carrefours des routes. Revue de l'ACR $9: 15-16$

39. ACR (1934) Les arbres sur le bord des routes sont-ils désirables? Sont-ils dangereux pour les automobilistes? Revue de l'ACR 4: $75-77$.

40. (1928) La route et les riverains agriculteurs. L'union du sud-est: 286,339

41. ACR (1953) Les arbres de nos routes ne sont pas des assassins. Revue de l'ACR 20-21:31.

42. CAQ (1930) Les routes du Québec. Service: 9.

43. Ministère de la Voirie de Québec [M. V. Qc.] (1926) Rapport du ministère de la Voirie. Imprimeur de sa Majesté le Roi, Québec.

44. CAQ (Club Automobile de Québec) (1924) La protection des arbres le long des routes. Motor Magazine: 15

45. Ministère de la Voirie de Québec [M. V. Qc.] (1930) Rapport du ministère de la Voirie. Imprimeur de sa Majesté le Roi, Québec.

46. Dupont J-C (1979) L'artisan forgeron. PUL, Québec.

47. Miraucourt S (1983) Histoire de la pompe à essence in « $\mathrm{La}$ mesure de la vie quotidienne », Culture technique 9:170-177.

48. (1956) Indicateur lyonnais Henry : annuaire commercial, administratif et judiciaire de la ville de Lyon et du département du Rhône, Lyon.

49. Augé M (1992) Non-lieux, introduction à une anthropologie de la surmodernité. Seuil, Paris.

50. Bertrand R (2011) L'Histoire à parts égales : Récits d'une rencontre Orient-Occident (XVI $-X V I I^{\mathrm{e}}$ siècle). Seuil, Paris.

51. Rosa H (2010) Accélération : une critique sociale du temps. La Découverte, Paris. 